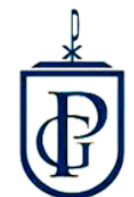

Veritas Et Scientia

Vol. $7, N^{\circ} 1,859-865$

Enero - Junio del 2018.

ISSN $2307-5139$

\title{
IDENTIFICACIÓN Y ANÁLISIS DE SÍNTOMAS MÚSCULO ESQUELÉTICOS DE ORIGEN DISERGONÓMICO EN EL PERSONAL DE ENFERMERÍA DEL HOSPITAL HIPÓLITO UNANUE DE TACNA - 2016
}

IDENTIFICATION AND ANALYSIS OF MUSCLE-SKELETAL SYMPTOMS OF DISERGONOMIC ORIGIN IN THE NURSING STAFF OF HIPÓLITO UNANUE DE TACNA HOSPITAL - 2016

Lourdes Amparo Cervantes Castillo ${ }^{1}$

Presentado: $10 / 02 / 2018$

Aceptado: $15 / 05 / 2018$

Publicado online: 11/06/2018

\section{RESUMEN}

Ésta investigación de campo en el área de la Salud Ocupacional tuvo como objetivo identificar y analizar los síntomas músculo-esqueléticos de origen disergonómico, en el Personal de Enfermería del Hospital Hipólito Unanue durante el año 2016.Estudio descriptivo, transversal, cuantitativo. Se identificó a 342 trabajadores del Departamento de Enfermería (43.86\% de personal profesional y el $56,14 \%$ técnicos de enfermería) a los cuales a través de una entrevista en su puesto de trabajo se aplicó el Cuestionario Nórdico de Kuorinka $\left(\mathrm{N}^{\circ} 2\right)$. Respecto a la percepción de la intensidad de dolor (escala de 1 a 5), los síntomas en Cuello, Codo o Antebrazo y Zona Dorsal o Lumbar se evalúan principalmente con una intensidad 3 , mientras que síntomas en codo-antebrazo y Muñeca o Mano con una intensidad 5. De acuerdo a la distribución según la duración de la molestia y por zona corporal; el $47 \%$ refiere que los síntomas le han durado de 1 a 7 días, donde las molestias fueron permanentes en la zona dorsal/lumbar $18.4 \%$, el $15 \%$ en hombro, y entre el $11 \%$ y $12 \%$ en cuello, codo, mano y/o muñeca. Cuando se presentaron éstos síntomas el $58 \%$ no necesitó ningún día de descanso médico, cerca del $35 \%$ requirió de 1 a 7 días, el $4.8 \%$ de 1 a 4 semanas y el $1.5 \%$ de más de un mes. Los trabajadores de los servicios de: Consultorios Externos y Hospitalización, presentan afecciones musculo-esqueléticas en la zona Dorsal o Lumbar, 58.02\% y $47.57 \%$ respectivamente, en el Hombro 35.00 \% y $27.67 \%$; en el Cuello $35.80 \%$ y $16.99 \%$; en la Muñeca o Mano $25.93 \%$ y 20.87 , respectivamente. En Cuidados Críticos los síntomas reportados se dan en la Zona

\footnotetext{
${ }^{1}$ Magíster en salud ocupacional y ambiental
} 
Dorsal y Lumbar $50.00 \%$ y en el Cuello $32.61 \%$. Finalmente, en el Servicio de Central de Esterilización, las molestias se dan principalmente en la Zona Dorsal o Lumbar $66.67 \%$ y en el Cuello $33.33 \%$. Los principales factores de riesgo disergonómico son: Movimiento o Posturas Dinámicas Forzadas 58.94\%, Posturas Estáticas Forzadas $38.14 \%$.

Palabras Clave: riesgo disergonómico, síntoma músculo-esquelético, personal enfermería.

\section{ABSTRACT}

This field research in the area of Occupational Health aimed to identify and analyze musculoskeletal symptoms of dysergonomic origin, in the Nursing Staff of Hipolito Unanue Hospital during 2016. Descriptive, cross-sectional, quantitative study. 342 workers of the Nursing Department were identified (43.86\% of professional staff and $56.14 \%$ nursing technicians) to whom the Kuorinka Nordic Questionnaire $\left(N^{\circ} 2\right)$ was applied through an interview at their workplace. Regarding the perception of pain intensity (scale from 1 to 5), the symptoms in Neck, Elbow or Forearm and Dorsal or Lumbar Zone are evaluated mainly with an intensity 3, while symptoms in elbowforearm and Wrist or Hand with an intensity 5. According to the distribution according to the duration of the discomfort and per body area; $47 \%$ reported that the symptoms lasted from 1 to 7 days, where the discomfort was permanent in the dorsal / lumbar area $18.4 \%, 15 \%$ in shoulder, and between $11 \%$ and $12 \%$ in neck, elbow, hand and / or doll. When these symptoms occurred, $58 \%$ did not need any medical rest day, about $35 \%$ required 1 to 7 days, $4.8 \%$ from 1 to 4 weeks and $1.5 \%$ more than a month. The workers of the services of: External Offices and Hospitalization, present muscular-skeletal affections in the Dorsal or Lumbar area, $58.02 \%$ and $47.57 \%$ respectively, in the Shoulder $35.00 \%$ and $27.67 \%$; in the Neck $35.80 \%$ and $16.99 \%$; in the Wrist or Hand $25.93 \%$ and 20.87 , respectively. In Critical Care the symptoms reported are in the Dorsal and Lumbar Zone $50.00 \%$ and in the Neck $32.61 \%$. Finally, in the Service of Central Sterilization, the discomfort occurs mainly in the Dorsal or Lumbar Area $66.67 \%$ and in the Neck $33.33 \%$. The main factors of disergonomic risk are: Movement or forced dynamic postures 58.94\%, Static Static Postures $38.14 \%$.

Keywords: disergonomic risk, musculoskeletal symptom, nursing.

\section{INTRODUCCIÓN}

Las principales Organizaciones Mundiales como la Organización Mundial de la Salud (OMS), la Organización Panamericana de la Salud (OPS), y la Organización Internacional del Trabajo (OIT), cada vez ponen especial énfasis en la necesidad de la vigilancia y control de los factores del medio ambiente y en especial en los centros laborales, como probables focos susceptibles de generar accidentes, enfermedades y malestar psicológico.En el Perú se promulgó la Ley 29783 de Seguridad y Salud en el Trabajo, consecuentemente se ha venido tomando conciencia sobre la importancia de la salud 
ocupacional no sólo como parámetro importante en la vigilancia de la salud pública, sino también por su importancia en el aparato productivo de cualquier empresa o institución y las relaciones laborales de las mismas. Actualmente las enfermedades profesionales y los accidentes de trabajo son responsables no sólo de elevadas pérdidas de índole económico, sino también pueden causar deterioro en la calidad de vida, dolor y sufrimiento en los trabajadores y sus familiares. Sin embargo, la sensibilización del público en general respecto de la seguridad y salud en el trabajo es muy escasa y con frecuencia no se le asigna la prioridad que merece (1). En la práctica, toda actividad laboral conlleva riesgos para la salud derivados de la presencia de factores físicos, químicos, biológicos, biomecánicos y psicosociales, causantes de accidente o de enfermedad (2). Estas enfermedades músculo esqueléticas derivadas de los riesgos ergonómicos son prevenibles, sin embargo ocasionan grandes pérdidas económicas por la disminución de la productividad y los costos requeridos para su atención (3). Los reportes de la OIT para el año 2012, el $25 \%$ de trabajadores reportaron algún tipo de patología de la columna cervical, dorsal y/o lumbar (4), la OMS reporta que el $80 \%$ de la población en algún momento de su vida ha padecido alguna dolencia músculo esquelética y el $37 \%$ presenta anualmente dorsalgias (5). En el Perú, según el reporte del CEPRIT Lima del 2012, el 96.4 \% de los trabajadores de la Administración Pública están expuestos a factores de riesgo disergonómico, de los cuales el 30.3\% han reportado algún tipo de patología (6). En nuestra Región Tacna, según el Informe del Análisis Situacional de Salud del 2013 reporta que las afecciones de este tipo son el $12 \%$ de todos los motivos de consulta en adultos (7), mientras que en ESSALUD son el $14 \%$ (8). A partir de la promulgación de la Ley 29783 de Seguridad y Salud en el Trabajo es imperativo que todas las instituciones Públicas y Privadas implementen Programas de Seguridad y Salud Ocupacional (9). La Salud Ocupacional busca la creación de ambientes de trabajo seguros y sanos, que permitan a los trabajadores conseguir un estado de salud pleno, alcanzando el bienestar físico, mental y social (10). Se denomina riesgo laboral a todo aquel aspecto del trabajo que tiene la potencialidad de causarle algún daño al trabajador (11) y pueden ser físico, mecánico, químico, biológico, psicosocial o ergonómico.

\section{OBJETIVOS}

- Identificar y cuantificar los síntomas y analizar algunas características clínicas músculo esqueléticos de origen disergonómico que presenta el Personal de Enfermería del Hospital Hipólito Unanue durante el año 2016.

- Identificar la etiología de los síntomas musculo-esqueléticos de origen disergonómico según características laborales del Personal de Enfermería y que presenta el Personal de Enfermería del Hospital Hipólito Unanue durante el año 2016.

\section{METODOLOGÍA}

Estudio descriptivo, transversal cuantitativo. Se realizó a partir del mes de enero del 2016, siendo el ámbito de investigación el Hospital de Apoyo Hipólito Unanue de Tacna. La población de estudio fue el personal del departamento de enfermería del Hospital Hipólito Unanue, compuesto por 150 Licenciados en Enfermería, 192 Técnicos de Enfermería y que cumplen los criterios de inclusión, estimando una población total de 342 trabajadores. Se aplicó el Cuestionario Nórdico de Kuorinka, estandarizado, validado y respaldado por el Consejo Nórdico de Ministros (12).

\section{RESULTADOS}

El $60.23 \%$ de la población labora en el servicio de Hospitalización, el $23.68 \%$ en el Servicio de Consultorios Externos, $13.45 \%$ en el Servicio de Cuidados Críticos y el $2.63 \%$ en Central de Esterilización. El $17.84 \%$ tienen menos de 10 años de antigüedad, un $20.47 \%$ de 10 a 19 años de servicio, un $31.58 \%$ 
entre 20 a 29 años, $28.65 \%$ entre 30 y 39 años. Sólo el $1.46 \%$ tiene 40 o más años de servicio, también podemos concluir que casi el $62 \%$ tiene más de 20 años de trabajo.

El 55.6\% de los trabajadores de enfermería refiere haber percibido síntomas en las zonas Dorsales y/o lumbares; el $32,2 \%$ menciona tener síntomas en el hombro, el $28.7 \%$ en el cuello, el $24,6 \%$ en la muñeca y/o mano y sólo el $11.1 \%$ en el codo y/o antebrazo (Tabla 01).

Tabla 1. Percepción de síntomas músculo-esqueléticos por zona corporal,

\begin{tabular}{|c|c|c|c|c|c|c|c|c|c|c|c|c|}
\hline \multirow{3}{*}{$\begin{array}{l}\text { P1: ¿¿Ha tenido } \\
\text { molestias en ...? }\end{array}$} & \multicolumn{10}{|c|}{ Respuestas } & \multirow{2}{*}{\multicolumn{2}{|c|}{ TOTAL }} \\
\hline & \multicolumn{2}{|c|}{ SI } & \multicolumn{2}{|c|}{ No } & \multicolumn{2}{|c|}{ IZQUIERDA } & \multicolumn{2}{|c|}{ DERECHA } & \multicolumn{2}{|c|}{ AMBAS } & & \\
\hline & $\mathbf{N}^{\circ}$ & $\%$ & $\mathbf{N}^{\circ}$ & $\%$ & $\mathbf{N}^{\circ}$ & $\%$ & $\mathbf{N}^{\circ}$ & $\%$ & $\mathbf{N}^{\circ}$ & $\%$ & $\mathbf{N}^{\circ}$ & $\%$ \\
\hline Cuello & 98 & $28.7 \%$ & 244 & $71.3 \%$ & 0 & $0.0 \%$ & 0 & $0.0 \%$ & 0 & $0.0 \%$ & & $100.0 \%$ \\
\hline Hombro & 110 & $32.2 \%$ & 232 & $67.8 \%$ & 28 & $8.2 \%$ & 72 & $21.1 \%$ & 10 & $13.9 \%$ & 342 & $100.0 \%$ \\
\hline Dorsal o Lumbar & 190 & $55.6 \%$ & 152 & $44.4 \%$ & 0 & $0.0 \%$ & 0 & $0.0 \%$ & 0 & $0.0 \%$ & 342 & $100.0 \%$ \\
\hline Codo o Antebrazo & 38 & $11.1 \%$ & 304 & $88.9 \%$ & 5 & $1.5 \%$ & 29 & $8.5 \%$ & 4 & $13.8 \%$ & 342 & $100.0 \%$ \\
\hline Muñeca o Mano & 84 & $24.6 \%$ & 258 & $75.4 \%$ & 11 & $3.2 \%$ & 54 & $15.8 \%$ & 19 & $35.2 \%$ & 342 & $100.0 \%$ \\
\hline
\end{tabular}

Fuente: Encuesta de Investigacion

De los resultados, según tiempo de molestias, el personal presenta estos síntomas menos de 1 mes (2.18\%), 22\% de 1 mes a un año y más de 1 año $76 \%$. En el $13 \%$ fue necesario el cambio de puesto de trabajo, el $21.1 \%$ de los casos por síntomas en Codo o Antebrazo, 14.3\% en Mano o Muñeca, $11.1 \%$ por síntomas en la Zona Dorsal o Lumbar, $10.2 \%$ en el Cuello y $8.2 \%$ en el Hombro. Y el $87.02 \%$ de casos no fue necesario el cambio de puesto de trabajo.

Al analizar la distribución según el tiempo que la sintomatología referida le impidió realizar su trabajo en los últimos doce meses, el $58 \%$ en promedio no necesitó ningún día, cerca del $36 \%$ necesitó de 1 a 7 días, el $4.8 \%$ de 1 a 4 semanas y sólo el 1.5\% necesitó más de un mes (Tabla 02).

Tabla 02. Tiempo que le impidió realizar su trabajo por zona afectada en los últimos 12 meses

\begin{tabular}{|c|c|c|c|c|c|c|c|c|c|c|}
\hline \multirow{3}{*}{$\begin{array}{l}\text { P7: ¿Cuánto tiempo estas } \\
\text { molestias le han } \\
\text { impedido hacer su trabajo } \\
\text { en los ultimos } 12 \text { meses? }\end{array}$} & \multicolumn{8}{|c|}{ Respuestas } & \multirow{2}{*}{\multicolumn{2}{|c|}{ TOTAL }} \\
\hline & \multicolumn{2}{|c|}{0 DIAS } & \multicolumn{2}{|c|}{ 1-7 DIAS } & \multicolumn{2}{|c|}{ 1- 4 SEMANAS } & \multicolumn{2}{|c|}{$>1$ MES } & & \\
\hline & $\mathbf{N}^{\circ}$ & $\%$ & $\mathbf{N}^{\circ}$ & $\%$ & $\mathbf{N}^{\circ}$ & $\%$ & $\mathbf{N}^{\circ}$ & $\%$ & $\mathbf{N}^{\circ}$ & $\%$ \\
\hline Cuello & 51 & $62.2 \%$ & 25 & $30.5 \%$ & 5 & $6.1 \%$ & 1 & $1.2 \%$ & 82 & $100.0 \%$ \\
\hline Hombro & 50 & $49.5 \%$ & 42 & $41.6 \%$ & 7 & $6.9 \%$ & 2 & $2.0 \%$ & 101 & $100.0 \%$ \\
\hline Dorsal o Lumbar & 84 & $48.3 \%$ & 80 & $46.0 \%$ & 7 & $4.0 \%$ & 3 & $1.7 \%$ & 174 & $100.0 \%$ \\
\hline Codo o Antebrazo & 22 & $61.1 \%$ & 11 & $30.6 \%$ & 2 & $5.6 \%$ & 1 & $2.8 \%$ & 36 & $100.0 \%$ \\
\hline Muñeca o Mano & 48 & $68.6 \%$ & 21 & $30.0 \%$ & 1 & $1.4 \%$ & 0 & $0.0 \%$ & 70 & $100.0 \%$ \\
\hline Promedio & & $57.9 \%$ & & $35.7 \%$ & & $4.8 \%$ & & $1.5 \%$ & & $100.0 \%$ \\
\hline
\end{tabular}

El $54 \%$ no recibió tratamiento alguno, y el $46 \%$ sí, siendo las molestias en la Zona Dorsal y/o Lumbar las que han sido tratadas en el $58 \%$ de los casos, hombro $44.6 \%$, codo o antebrazo en $44.4 \%$, muñeca o mano en $42.9 \%$ y cuello en $40.2 \%$. el $44.4 \%$ de los casos lo califican con una intensidad de 3 , siendo los más significativos los síntomas de cuello, hombro y los dorso-lumbares. Sólo el $6 \%$ los evalúa con una intensidad de 5 , fundamentalmente los que padecen de síntomas de codo y/o antebrazo, muñeca y mano. Por otro lado, poco más de $20.54 \%$ los evalúan con una intensidad leve ( 1 y 2 ) (Tabla 03). 
Tabla 03. Intensidad por zona afectada

\begin{tabular}{|c|c|c|c|c|c|c|c|c|c|c|c|c|}
\hline \multirow{3}{*}{$\begin{array}{l}\text { P10: Pongale nota a sus } \\
\text { molestias entre } 1 \text { (sin } \\
\text { molestias) y } 5 \\
\text { (molestias muy fuertes) }\end{array}$} & \multicolumn{10}{|c|}{ RESPUESTAS } & \multirow{2}{*}{\multicolumn{2}{|c|}{ TOTAL }} \\
\hline & \multicolumn{2}{|r|}{1} & \multicolumn{2}{|r|}{2} & \multicolumn{2}{|r|}{3} & \multicolumn{2}{|r|}{4} & \multicolumn{2}{|r|}{5} & & \\
\hline & $\mathbf{N}^{\circ}$ & $\%$ & $\mathbf{N}^{\circ}$ & $\%$ & $\mathbf{N}^{\circ}$ & $\%$ & $\mathbf{N}^{\circ}$ & $\%$ & $\mathbf{N}^{\circ}$ & $\%$ & $\mathbf{N}^{\circ}$ & $\%$ \\
\hline Cuello & 8 & $9.8 \%$ & 20 & $24.4 \%$ & 48 & $58.5 \%$ & 4 & $4.9 \%$ & 2 & $2.4 \%$ & 82 & $100.0 \%$ \\
\hline Hombro & 11 & $10.9 \%$ & 38 & $37.6 \%$ & 42 & $41.6 \%$ & 7 & $6.9 \%$ & 3 & $3.0 \%$ & 101 & $100.0 \%$ \\
\hline Dorsal o Lumbar & 10 & $5.7 \%$ & 37 & $21.3 \%$ & 97 & $55.7 \%$ & 20 & $11.5 \%$ & 10 & $5.7 \%$ & 174 & $100.0 \%$ \\
\hline Codo o Antebrazo & 1 & $2.8 \%$ & 17 & $47.2 \%$ & 12 & $33.3 \%$ & 2 & $5.6 \%$ & 4 & $11.1 \%$ & 36 & $100.0 \%$ \\
\hline Muñeca o Mano & 8 & $11.4 \%$ & 24 & $34.3 \%$ & 23 & $32.9 \%$ & 10 & $14.3 \%$ & 5 & $7.1 \%$ & 70 & $100.0 \%$ \\
\hline Promedio & & $8.1 \%$ & & $33.0 \%$ & & $44.4 \%$ & & $8.6 \%$ & & $5.9 \%$ & & $100.0 \%$ \\
\hline
\end{tabular}

Los principales factores de riesgo disergonómico que identificaron los trabajadores como causantes de las molestias son: Movimiento o Posturas Dinámicas Forzadas 58.94\%, Posturas Estáticas Forzadas $38.14 \%$. Del mismo modo se menciona a factores sicosociales como el estrés, y al clima factor no disergonómico. Los trabajadores de los Servicios de Consultorios Externos presentan afecciones músculo-esqueléticas principalmente en la zona Dorsal o Lumbar $58.02 \%$ y en hombro $41.98 \%$. En hospitalización $47.57 \%$ en la zona dorso lumbar y $27.67 \%$ en el hombro. En Cuidados Críticos los síntomas reportados se dan mayoritariamente en la Zona Dorsal y Lumbar $50.00 \%$ y en el Cuello $32.61 \%$. Finalmente, en el Servicio de Central de Esterilización, las molestias registradas también se dan principalmente en la Zona Dorsal o Lumbar $66.67 \%$ y en el Cuello $33.33 \%$.

\section{DISCUSIÓN}

De la evaluación realizada se concluyó la existencia de síntomas musculo-esqueléticos de origen disergonómico en ambos grupos ocupacionales. El personal de Enfermería ha percibido molestias en el $55.6 \%$ en la zona dorso-lumbar, el $32,2 \%$ en hombro, $28.7 \%$ en cuello, $24.6 \%$ en mano y $11.1 \%$ en codo; predominando el lado derecho en hombro y muñeca o mano haciendo más difícil sus tareas por ser el miembro superior dominante; y en ambas sobre todo en muñeca o mano con un $35.2 \%$. Según la antigüedad, observamos que el mencionado personal presenta síntomas en promedio $2.18 \%$ menos de un mes, $22 \%$ de un mes a un año, y $76 \%$ más de 1 año, por lo que concluimos que son dolencias crónicas que ocasionan episodios de dolor insidioso. En México, Barragan H. (2006) muestra asociación e interacciones entre la lumbalgia invalidante y variables laborales como: puesto de trabajo, antigüedad, esfuerzo físico y levantamiento de cargas (13). Leal, E. Mejías, H. y Pernalete, C (2001) encontraron la relación entre la sobrecarga física de trabajo y las dolencias músculo - esqueléticas presentes en el personal de enfermería de la Unidad Clínica de Cirugía y Traumatología del Hospital General Guatire Guarenas (14). En Venezuela Muñoz, A y Rodríguez, C (2001) demostró que las lesiones dorso-lumbares están relacionadas con la sobrecarga física de trabajo aplicada durante la jornada laboral (15). Curro 0 , Pecho M, Loza V, Ica (2009), en un estudio realizado entre enfermeras en la Región Ica, se encontró que el $26.2 \%$ de las enfermeras del MINSA presentan dolor leve de espalda al permanecer de pie por más de 06 horas, mientras que en Es-Salud fueron el 39,3\%. En los Hospitales del MINSA manifestaron dolor dorsal leve al manipular cargas superiores a $15 \mathrm{Kg}$ en el 20,7\% y en Es-Salud fue el 35,9\%. Las enfermeras encuestadas del MINSA y Es-SALUD, presentaron dolor cervical leve al movilizar pacientes, siendo los resultados a nivel del MINSA un porcentaje de 30,3\% de 44 y en Es-SALUD 32,4\% (16).

Ruiz F, Palomino J, Zambrano R, Llap C, (2003), realizaron el estudio “Prevalencia, impacto en la productividad y costos totales de las principales enfermedades en los trabajadores de un hospital al sur del Perú en el año 2003" se encontró que las enfermedades de la columna vertebral aquejan al 36.73\% 
de todos los trabajadores del hospital, siendo además la principal causa de ausentismo laboral los trastornos músculo esqueléticos (17). Ramirez - Segura C, Montenegro $M$, elaboraron el estudio "Condiciones de trabajo y perfil sanitario en los trabajadores de salud del Hospital Nacional Almanzor Aguinaga Asenjo - EsSalud. Chiclayo - 2009" se encontró que un 18,11\% presentó/a alguna enfermedad ocupacional, y con mayor frecuencia los problemas musculo-esqueléticos, cardiovasculares y problemas mentales (18). El estudio realizado por Rodríguez C mostró que un $62,9 \%$ de los trabajadores presentó problemas osteo-musculares (19), por las malas condiciones de diseño de las ambulancias que generaban estas enfermedades como dolor de espalda. Sus bajos niveles de ingresos les obligaban a desempeñar jornadas que iban más allá de sus capacidades. Además hacían trabajos nocturnos alterando su vida social - sentimental por que invertían su ritmo de vida respecto del grupo familiar.

Como podemos observar los resultados obtenidos en la presente investigación tienen correspondencia. Es la región dorso - lumbar, hombro y cuello la que se ve principalmente expuesta a dolencias de carácter músculo-esqueléticas. Del mismo modo, estos estudios coinciden en que las ocurrencias de estos se deben principalmente a sobrecarga física laboral, que predispone a los trabajadores a enfrentarse a los factores de riesgo de carácter disergonómico. La exposición a los mencionados factores se produce de la siguiente manera: el personal tiene principalmente peligros ergonómicos que provienen de Posturas Estáticas Forzadas y Posturas Dinámicas Forzadas, que a su vez se agravan por el Diseño Inadecuado de los Puestos de Trabajo. Del mismo modo pueden verse expuestos a aquellos que vienen como consecuencia de manipulación manual de cargas, y movimientos repetitivos inherentes a su formación ocupacional y asignación de funciones por tipo de puesto de trabajo. Una vez identificados los Síntomas Músculo Esqueléticos de origen disergonómico en el Personal de Enfermería del Hospital Hipólito Unanue es necesario implementar medidas de control que permitan corregir las deficiencias y de esta manera minimizar el nivel de los peligros y riesgos disergonómico y sus consecuencias como ausentismo laboral. El personal de Enfermería de los Servicios de: Consultorios Externos y Hospitalización; presentan síntomas músculo-esqueléticos principalmente en la zona Dorsal o Lumbar $58.02 \%$ y $47.57 \%$ respectivamente; en el Hombro $41.98 \%$ y $27.67 \%$. En Cuidados Críticos los síntomas reportados se dan mayoritariamente en la Zona Dorsal y Lumbar $50.00 \%$ y en el Cuello $32.61 \%$. Finalmente, en el Servicio de Central de Esterilización, las molestias registradas también se dan principalmente en la Zona Dorsal o Lumbar $66.67 \%$ y en el Cuello $33.33 \%$.

Ser recomienda implementar un monitoreo de factores ocupacionales de riesgo ergonómicos y análisis de puesto laboral, que permitan conocer la problemática más a fondo y que a su vez puedan facilitar otras acciones preventivas en beneficio de los trabajadores como el rediseño de puestos de trabajo. Se hace necesario la implementación de medidas preventivas (capacitación, pausa activa, mobiliario y equipo ergonómico, entre otras) que mitiguen los factores de daño. Se recomienda asimismo cumplir con la realización de los exámenes Médico Ocupacionales periódicos, en forma anual, sobre todo al personal con más de 50 años de edad y que supera los 20 años de servicio, porque el tiempo de exposición a los riesgos disergonómicos es mayor, ya que la actividad de prestación de Servicios de Salud es considerara de Alto Riesgo.

\section{REFERENCIAS BIBLIOGRÁFICAS}

1. Almenara Barrios J, Gonzales Caballero JL. Morbilidad hospitalaria de las personas mayores en Andalucía. Colección. Monografías de Investigación ed. Andalucía; 2000-2004.

2. DOCPLAYER. Programa SVE prevención de desórdenes músculo esqueléticos. [Online].; 2011 [cited 2016 Julio 17. Available from: http://docplayer.es/7398020-Programa-sve-prevencion-dedesordenes-musculo-esqueleticos.html.

3. Dirección Ejecutiva de Salud Ocupacional. Manual de Salud Ocupacional. Lima: Ministerio de Salud; 2005. 
4. Organización Internacional del Trabajo. Boletín Regional de América Latina y el Caribe, Seguridad y Salud en el Trabajo; 2015.

5. Organización Internacional del Trabajo, Organización Mundial de la Salud. Moving knowledge of global burden into preventive action. American Journal of Industrial Medicine. 2005 Noviembre; XLVIII: p. 395-399.

6. Centro de Prevención de Riesgo del Trabajo. EsSalud. Perfil de Morbilidad de los Trabajadores 2006-2009. Lima: EsSalud; 2012.

7. Dirección de Epidemiología, Dirección Regional de Salud Tacna. Análisis de la Situación de Tacna 2013. Tacna: Dirección Regional de Salud Tacna; 2014.

8. CEPRIT Tacna. EsSalud. Boletín Informativo: Riesgos ligados a condiciones ergonómicas. Tacna: EsSalud; 2015.

9 Ley de Seguridad y Salud en el Trabajo. Ley N²9783. Diario Oficial el Peruano. 2011 agosto 20.

10 Portal del Ministerio de Salud de Argentina. Buenos Aires: Ministerio de Salud. [Online].; 2005 [cited 2015 diciembre 10. Available from: http://www.msal.gob.ar/index.php/home/saludocupacional.

11 DefiniciónABC.com. Definición de Riesgo Laboral [sede Web]. [Online].; 2015 [cited 2016 octubre 15. Available from: http://www.definicionabc.com/salud/riesgo-laboral.php.

12 Kuorinka I, Jonsson B, Kilbom A, Vinterberg H, Biering-Sorensen F, Andersson G, et al. Standardised Nordic questionnaires for the analysis of musculoskeletal symptoms. Applied Ergonomics. 1987 September; XVIII(3): p. 233-237.

13 Barragán L. Cap. 17 Necesidades, demanda y oferta de Atención Médica. In Fundamentos de Salud Pública.:

http://inus.org.ar/documentacion/Documentos\%20Tecnicos/Fundamentos_de_la_salud_publica /cap_17.pdf p. 353-360.

14 Briceño G, Fagundez E, Quintero D. Alteraciones músculo-esqueléticas en la enfermera(o) quirúrgica(o) en el Hospital Militar 'Dr. Carlos Arvelo' durante el primer semestre de 2006. Tesis de Pregrado. Caracas: Universidad Central de Venezuela, Ciencias de la Salud; 2011. Report No.: M2006 B84.

15 Harari F. Cumulative Trauma Disorders in the Workplace. In Services DoHaH Services USDoHaH, editor. USA; 2009.

16 Curro O, Pecho M, Loza V, Carbonel T, Calle D. Riesgos ergonómicos de los profesionales de Enfermeria que laboran en las áreas críticas de los Hospitales del Minsa y ESSALUD del Departamento de Ica. ; 2009.

17 Ruiz Gutiérrez F, Palomino Baldeón J, Zambrano Beltrán R, Llap Yesán C. Prevalencia, impacto en la productividad y costos totales de las principales enfermedades en los trabajadores de un Hospital al Sur del Perú en el año 2003..

18 Ramírez-Segura CL, Montenegro Orrego M, Neciosup-Puican. Condiciones de trabajo y perfil sanitario en los trabajadores de salud del Hospital Nacional Almanzor Aguinaga Asenjo - EsSalud. Revista del Cuerpo Médico Hospital Nacional Almanzor Aguinaga Asenjo. 2009; VI(3): p. 17-21.

19 Rodríguez JC. Perfil de salud y condiciones de trabajo en personal masculino de enfermería en un servicio ambulante de atención de emergencias Venezuela; 2000. 